\title{
Characterization of the Physical - Mechanical Properties of Alpaca Fiber (Vicugna pacos) at the Tunshi Experimental Station
}

\section{Caracterización de las Propiedades Físico - Mecánicas de la Fibra de Alpaca (Vicugna pacos) de la Estación Experimental Tunshi}

VII International Congress of Science, Technology, Entrepreneurship and Innovation (SECTEI 2020)

Corresponding Author:

Maritza L. Vaca-Cardenas

maritza.vaca@espoch.edu.ec

Published: 26 August 2021

Production and Hosting by Knowledge $E$

(c) Maritza L. Vaca-Cardenas et al. This article is distributed under the terms of the Creative Commons Attribution License, which permits unrestricted use and redistribution provided that the original author and source are credited.

\section{Maritza L. Vaca-Cardenas, M. Oleas, Mónica Elva Vaca-Cárdenas, and A. Velasco}

Docente Facultad de Ciencias Pecuarias, Escuela Superior Politécnica de Chimborazo, Riobamba, Ecuador

\section{Abstract}

The alpaca is one of the four South American camelids that mainly inhabit the inter-Andean zone of Ecuador. Alpaca fiber is characterized by being a natural, soft and resistant fiber, of which, the fleece is the most valued part. The objective of this research was to evaluate the quality parameters of alpaca fiber in terms of physical-mechanical properties. The research was carried out in the Tunishi Experimental Station, ESPOCH. A descriptive statistic of mean, minimum and maximum range, and separation of means per student was applied. The alpaca with characteristics of Huacaya breed was selected and the shearing was carried out manually to obtain the fleece. Later, the performance of the fleece was evaluated, which was $85.71 \%$. Two categories of fiber were selected: fine and thick. Sixty samples were taken for analysis by stretched and unstretched fiber length. The measurements of unstretched fiber length in $\mathrm{cm}$ for the thin and thick fiber were $12.50 \mathrm{~cm}$ and $13.52 \mathrm{~cm}$ respectively, presenting significant differences $(p \leq 0.05)$. The measurements of the stretched fiber length in $\mathrm{cm}$ were $17.29 \mathrm{~cm}$ for fine fiber and $17.27 \mathrm{~cm}$ for thick fiber, presenting no significant differences $(p \geq 0.05)$. The resistance of thread and fabric for fine fiber was $590 \mathrm{~N} / \mathrm{cm}^{2}$, and for thick fiber was 2835.5 $\mathrm{N} / \mathrm{cm}^{2}$, presenting highly significant differences $(p \leq 0.01)$. Regarding the percentage of yarn elongation, the values obtained were $19 \%$ for fine fiber and $12 \%$ for thick fiber, observing highly significant differences $(p \leq 0.01)$. The thick fiber fabric had a better resistance ( $2.3 \mathrm{BAR})$ than the fine fiber fabric (1.7 BAR), with a time of $2.34 \mathrm{~s}$ and $1.88 \mathrm{~s}$ respectively. The classification of the fiber by its softness did not present significant differences. Finally, regarding the sensory classification, it was established as a soft fiber with $91 \%$.

Keywords: alpaca, fleece, fine fiber, thick fiber, fiber properties.

\section{Resumen}

La alpaca es uno de los cuatro camélidos sudamericanos que principalmente habitan en la zona interandina del Ecuador. La fibra de alpaca se caracteriza por ser una fibra natural, suave y resistente; de la cual, el vellón es la parte de la fibra del animal más valorada. El objetivo de esta investigación fue evaluar los parámetros de calidad de fibra de alpaca en cuanto a las propiedades físico - mecánicas. La investigación se la realizó en la Estación Experimental TUNSHI - ESPOCH. Se aplicó una estadística descriptiva de media, rango mínimo y máximo y separación de medias por t estudent. Se seleccionó a la alpaca con características de la raza Huacaya y se realizó la esquila por el método manual para la obtención del vellón. Posteriormente, se evaluó el rendimiento del vellón el cual fue de $85,71 \%$, se seleccionaron 
dos categorías de fibra fina y gruesa. Sesenta muestras fueron tomadas para su análisis: longitud de mecha estirada y sin estirar. La media de longitud de fibra sin estirar en $\mathrm{cm}$ para la fina y gruesa fueron de 12,50 y 13,52 correspondientemente, presentando diferencias significativas $(p \leq 0,05)$, La media de la longitud en $\mathrm{cm}$ de fibra estirada determinó que la fibra fina presentó una media de 17,29 y la fibra gruesa de $17,27 \mathrm{~cm}$, sin presentar diferencias significativas $(p \geq 0,05)$. La resistencia del hilo y tejido para la fibra fina fue de $590 \mathrm{~N} / \mathrm{cm}^{2}$ y para la gruesa fue de $2835,5 \mathrm{~N} / \mathrm{cm}^{2}$, presentando diferencias altamente significativas $(p \leq 0,01)$. Finalmente, en cuanto al porcentaje de elongación de hilo, presentó un 19\% para fibra fina y un $12 \%$ para gruesa, observándose también diferencias altamente significativas $(p \leq 0,01)$. E tejido de la fibra gruesa tuvo mejor resistencia (2,3 BAR) que el de la fibra fina (1,7 BAR), con un tiempo de 2,34 s y 1,88 s respectivamente. La clasificación de la fibra por su suavidad, no presentó diferencias significativas. En cuanto a la clasificación sensorial se la ubico como una fibra suave con el $91 \%$.

Palabras Clave: Alpaca, Vellón, Fibra fina, Fibra gruesa, Propiedades de la fibra.

\section{Introducción}

La alpaca, es uno de los camélidos sudamericanos, que en la actualidad no es considerada un animal de carga o de trabajo, sino que se encuentra en estado doméstico criada en rebaños. Su principal actividad económica es la producción de fibra para la obtención de diversos artículos textiles [1].

En referencia a los países productores de fibra de alpaca, Perú es el principal productor, con una población de 3685516 alpacas [2]. El Ecuador en el año 2000 tuvo una población de 2024 alpacas y para el 2014 llego a 6595 semovientes [3]. La provincia de Chimborazo cuenta con 346 alpacas de acuerdo al III Censo Nacional Agropecuario [3]. El Ministerio de Agricultura Ganadería Acuacultura y Pesca en el año 2013 importó 200 alpacas desde Perú, con la finalidad de mejorar la calidad genética de las caravanas del país, en beneficio de los pequeños productores a cargo de estas especies.

La especie alpaca se caracteriza por tener dos razas las cuales son: Suri y Huacaya. En la industria textil ecuatoriana la fibra de alpaca que se utiliza es la que proviene de la raza huacaya, debido a su fácil empleo para la obtención de su fibra. Esta especie se desarrolla en lugares de gran altura como los páramos, cerros, partes húmedas etc. La raza huacaya se rige por condiciones climáticas desfavorables, donde la principal fuente de alimento son las hierbas y se caracterizan por tener una fibra abundante y de crecimiento perpendicular, con aspecto esponjoso y más corta en comparación a la raza suri [4]. 
La fibra de alpaca es el producto utilizado en la fabricación de diversas prendas textiles por su excelente calidad y ocupa una importante demanda a nivel mundial. La fibra de la alpaca huacaya es $100 \%$ natural cuyo crecimiento anual es de 9 a $12 \mathrm{~cm}$ de diámetro [5]. Para la cosecha de fibra, la esquila es una actividad muy importante dentro del programa de manejo de alpacas. La esquila es un proceso que consiste en cortar y separar con la utilización de tijeras o maquinas especiales la fibra del animal el cual puede ser alpaca, llama, ovino, etc. Con la finalidad de obtener el vellón y con el cuidado máximo posible para evitar la ruptura entre ellas.

La esquila de la alpaca esta en función al calendario alpaquero y se toma en consideración los meses de menor lluvia durante la época de verano debido a la sensibilidad de la fibra, ya que esta no debe mojarse porque pierde sus propiedades y por ende su calidad. Si se realiza la esquila durante la época de lluvia es necesario que las alpacas estén cubiertas por cobertizos para mantener seca la fibra [6]. El vellón es la fibra que cubre al animal el cual se clasifican de acuerdo con su calidad en dos: el manto es la parte de mayor calidad y se ubica en el lomo, costillar y paletas del animal y las bragas son las fibras de menor calidad ya que son mucho más cortas, gruesas y sucias además, y se ubican en las patas y el cuello.

\section{Metodología}

\subsection{Localización y Duración del Experimento}

La presente investigación se realizó en el área de producción de camélidos sudamericanos de la Estación Experimental Tunshi de la Facultad de Ciencias Pecuarias de la ESPOCH, ubicado en el KM 12 vía a Licto, cantón Riobamba, Provincia Chimborazo. La duración de la investigación fue de 120 días (4 meses) los cuales fueron distribuidos conforme a las necesidades de tiempo para cada actividad a partir de la selección de los animales antes de realizarse la esquila. El análisis de las muestras se lo efectuó en el laboratorio de fibras agroindustriales de la Facultad de Ciencias Pecuarias de la ESPOCH.

\subsubsection{Unidades experimentales}

El presente trabajo es de tipo descriptivo, no presenta tratamientos se tomaron 60 muestras de fibra del costillar o costado del vellón, de acuerdo a lo establecido por Aylan-Parker y McGregor [7], las cuales posteriormente fueron los objetos de estudio. 


\subsubsection{Mediciones experimentales}

- Rendimiento del vellón

- Longitud de fibra sin estirar

- Longitud de fibra estirada

- Porcentaje de elongación (\%)

- Resistencia a la tensión (N/cm2)

\subsubsection{Materiales}

- Mandil

- Tijeras de esquila

- Saquillos de yute

- Fundas plásticas

- Estacas

- Sogas

- Regla en centímetros

- Cartulina de color negro

- Lupa

\subsubsection{Equipos}

- Cámara fotográfica

- Elastómero

- Lastometro

- Balanza

\subsubsection{Instalaciones}

- Unidad Experimental Tunshi área de camélidos sudamericanos

- Laboratorio de lanas y fibras de la Facultad de Ciencias Pecuarias

\subsubsection{Procedimiento}

La esquila se la realizó de manera tradicional. La estación cuenta con alpacas de la raza huacaya para lo cual se seleccionó una de color blanco y se trasportó el animal 
desde el lugar de reposo hacia el lugar de la esquila con mucho cuidado. Se efectuó una breve limpieza del vellón antes de entrar al lugar de esquila. Se determinó el largo de mecha de la fibra observando el costillar para ver su longitud, con ayuda de una regla obteniendo como resultado que tuvo una longitud de mecha de $17 \mathrm{~cm}$.

Posteriormente se procedió con el tumbado por el método de extremidad contraria, sujetando de la cabeza al animal y se cubrió los ojos para evitar el estrés del mismo. A continuación, se sujetaron las patas del otro lado y se haló a su parte contraria provocando el desequilibrio del animal y por ende su caída. Se extendió al animal de costado sobre un saquillo de yute y fundas plásticas y se sujetó sus extremidades (patas) con la ayuda de sogas que se amarró a unas estacas. Entonces, se empezó a esquilar por la parte del pecho y vientre hacia la espalda, incluyendo el cuello del lado derecho y las bragas (patas y cabeza). Luego se dio la vuelta al animal y se realizó lo mismo con el lado izquierdo. Los cortes se realizaron con la tijera de forma adecuada y uniforme, siguiendo un orden. Se evitó en todo momento el doble corte de esta manera se evita tener fibras pequeñas.

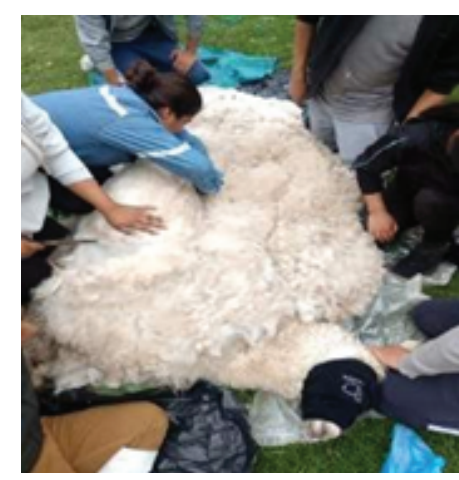

Figure 1

Esquila de Alpaca.

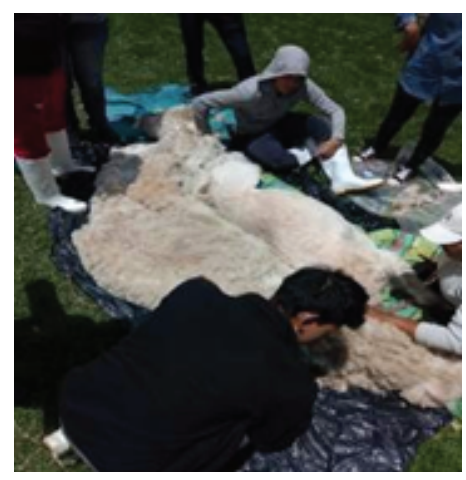

Figure 2

Esquila lado izquierdo. 
Terminada la esquila se soltó al animal de manera cuidadosa sin que pise el vellón y se procedió a retirar las impurezas y a clasificar la fibra de bragas y manto. Seguidamente el vellón se dobló a partir de la parte del lomo del animal, se juntó cara con cara, sin que se contamine con los pelos o alguna materia extraña al vellón. Es decir, la parte externa del vellón se dejó afuera y la parte más limpia del vellón debe dejo hacia adentro.

Luego se procedió a envellonar a partir del vellón doblado, se inició de la parte posterior hacia el cuello; después, el vellón de la región del cuello se torció como una especie de soga para con ello asegurar el vellón enrollado a manera de tambor. Luego del envellonado de la fibra se realizó el pesado del vellón completo para la cual se usó una balanza. El peso fue de 3,5 lb, y el peso solo del manto fue de 1,3 lb y las bragas tuvo un peso de 1,9 lb libras. Por último, se realizó el pesado de la basura como tierra, pasto, etc., que fue de 0,3 lb. Finalmente, se calculó el rendimiento al lavado de la fibra.

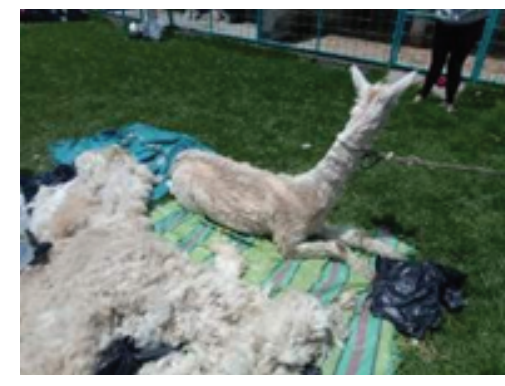

Figure 3

Vellón de Alpaca.

Para el proceso del lavado se remojó la fibra en una olla con agua caliente en la cual se colocó detergente para dejarlo reposar por 2 hr, luego se procedió al lavado con agua fría hasta quitar todo el detergente y se procedió a colocar la fibra en un tendedero para el secado, una vez que se encontró seco se realizó el deserdado y el escarmenado de la fibra. Una vez que se realizó el deserdado y el escarmenado se procedió al hilado y tejido de la fibra para la posterior elaboración de una prenda de vestir.

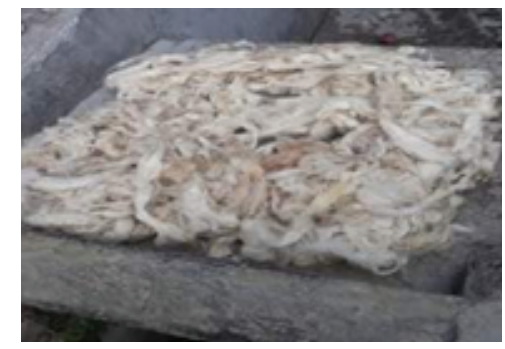

Figure 4

Lavado de la fibra. 


\subsubsection{Pruebas físicas de laboratorio}

Se analizaron $5 \mathrm{~g}$ de fibra de cada parte del animal de fibra fina (costillas) y gruesa (bragas) para el respectivo estudio de numero de rizos/cm, longitud de mecha con y sin estirar por $\mathrm{cm}$.

Para el conteo de rizos se tomó 60 muestras de fibra fina y 60 muestras de fibra gruesa y se utilizó una cartulina negra para poder observar las ondulaciones, la mecha y con ayuda de una lupa se realizó el conteo de las ondulaciones.

Para determinar la longitud de fibra se utilizó dos pinzas, se sujetó los extremos de la fibra y con la implementación de una regla, se midió la longitud, sin estirar y estirada tanto en la fibra gruesa como en la fibra fina donde se obtuvo un total de 120 muestras. Posteriormente, se realizó la prueba T de Student.

Las pruebas de resistencia del hilado y del tejido de la fibra se realizó en el laboratorio de fibras y lanas de la FCP en donde se utilizó el elastómero y el lastometro. Dichas pruebas se realizaron con 50 muestras de hilo de fibra fina y 50 muestras de fibra gruesa y una muestra de tejido de fibra fina y una muestra de tejido de fibra gruesa de alpaca huacaya.

\subsubsection{Pruebas de análisis sensorial}

Se realizó las pruebas de suavidad al tacto en el laboratorio de fibras de la FCP en donde al azar se escogió a 11 estudiantes los mismos que emplearon los órganos de los sentidos como la vista y tacto, para su posterior prueba de ordenamiento de fibra más fina a la más gruesa.

\section{Resultados y Discusión}

\subsection{Rendimiento del Vellón al Lavado}

El vellón fue clasificado de acuerdo a la Norma Técnica Ecuatoriana NTE INEN 2852.2015 [8].

El rendimiento al lavado del vellón se basó en el método IWTO - 19 [9], que consistió en el desengastado y remoción de materias extrañas (suciedad), cuidando la perdida de fibra.

\section{Rendimiento del Vellón:}

$$
R \frac{W \text { fibra limpia }}{W \text { fibra sucia }} \times 100
$$




\section{Table 1}

Rendimiento del vellón.

\begin{tabular}{l|l} 
Fibra sucia & \\
\hline Datos & Peso \\
\hline Fibra fina & $1,3 \mathrm{Ib}$ \\
\hline Fibra gruesa & $1,9 \mathrm{Ib}$ \\
\hline Basura & $0,3 \mathrm{lb}$ \\
\hline Peso del vellón & $3,5 \mathrm{Ib}$ \\
\hline
\end{tabular}

\begin{tabular}{l|l}
\hline Fibra limpia & Pesos \\
\hline Datos & $0,9 \mathrm{lb}$ \\
\hline Fibra fina & $1,4 \mathrm{lb}$ \\
\hline Fibra gruesa & $0,7 \mathrm{lb}$ \\
\hline Cerda & $3,0 \mathrm{lb}$
\end{tabular}

$$
\begin{gathered}
R \frac{3,0 l b}{3,5 l b} \times 100 \\
R=85,71 \%
\end{gathered}
$$

El rendimiento al lavado de la fibra de alpaca Huacaya fue $85,71 \%$, Estudios similares como el de Villaroel (1959) reportó resultados de 90,3\%, con rango entre 86,6 y 93,7 \%. En de 287 alpacas de Puno (Perú), reportaron un rendimiento promedio de 87,44\% con un rango entre 86,54 y 89,52\%; mientras que Wuliji et al. [10], Aylan-Parker y McGregor [7], McGregor [11] y Lupton et al. (2006b), encontraron valores entre 89 a 95\% para alpacas en Nueva Zelanda, Australia y E.E.U.U, respectivamente. Consecuentemente, los hallazgos de este estudio se asemejan a lo reportado por la mayor parte de los autores citados.

Posteriormente se efectuó al hilado en una máquina automática que facilite el proceso de hilado de fibra, en especial el hilado de fibra de alpaca.

\subsection{Longitud de la Fibra sin Estirar}

En la Tabla 2 se observa la media de longitud de fibra tanto para la fibra fina como la gruesa, teniendo una media de 12,50 y $13,52 \mathrm{~cm}$ respectivamente presentando diferencias significativas $(p \leq 0,05)$. En cuanto a la longitud la fibra fina presenta un rango promedio de longitud de $17 \pm 6$ mientras que la fibra gruesa presenta un rango promedio de longitud de $20,5 \pm 6$ con una desviación estándar de 2,39 para la fibra fina y 3,05 para la fibra gruesa.

Por lo que se puede determinar que la fibra gruesa presentó valores más altos a los reportados por Montesinos, R (2000) al evaluar la fibra Huacaya encontró un promedio de 13,32 cm. Mientras que la fibra fina se encuentra por debajo de este. Sin embargo, estos resultados no son muy alejados del promedio citado.

Por otro lado, Gamarra Sánchez [12] afirma que la Longitud de fibra en las fibras de alpacas tienen una media de $12,77 \pm 1,37 \mathrm{~cm}$. 
Table 2

Longitud de fibra sin estirar.

Longitud sin estirar
Media
Varianza
Desviación estándar
Observaciones
Mínimo
Máximo
Coeficiente de correlación de
Pearson
Diferencia hipotética de las
medias
Grados de libertad
Estadístico t
P(T <= t) una cola

\begin{tabular}{ll} 
Fina & Gruesa \\
\hline 12,5033333 & 13,52416667 \\
5,73964972 & 9,33097387 \\
2,395756606 & 3,054664281 \\
60 & 60 \\
6 & 6 \\
17 & 20,5 \\
\hline & $-0,002269311$ \\
& 0 \\
\hline & 59 \\
& 2,034636375 \\
& 0,023194714
\end{tabular}

\subsection{Longitud de Fibra Estirada}

Table 3

Longitud de fibra estirada.

Longitud estirada
Media
Varianza
Desviación estándar
Observaciones
Mínimo
Máximo
Coeficiente de correlación de
Pearson Diferencia hipotética de
las medias
Grados de libertad
Estadístico t
$\mathrm{P}(\mathrm{T}<=\mathrm{t})$ una cola

Fina
17,2983333
4.485929379
2,11800127
60
13,4
21

\begin{tabular}{l} 
Gruesa \\
17,27 \\
4,847220339 \\
2,201640375 \\
60 \\
13 \\
22,2 \\
0.0629430880 \\
\hline 59 \\
-0.074210484 \\
0.470546971 \\
\hline
\end{tabular}

La longitud de fibra estirada presento una media de $17,29 \mathrm{~cm}$ para la fibra fina y de $17,27 \mathrm{~cm}$ para la fibra gruesa. Con un mínimo de 13,40 y un máximo de $21 \mathrm{~cm}$ y de 13 y $22,2 \mathrm{~cm}$ para la fibra gruesa. Valores que tiene relación con la fibra sin estirar. 
Table 4

Resistencia a la tensión (N/cm2).

Datos
Media
Mínimo
Máximo
Varianza
Desviación estándar
Coeficiente de variación
$\mathrm{P}(\mathrm{T}<=\mathrm{t})$ una cola

Fibra fina
$590 \mathrm{~N} / \mathrm{cm} 2$
250
862,5
26843,1122
163,838677
0,07198498
$7,11105488375584 \mathrm{E}-34$

Fibra Gruesa
$2835,5 \mathrm{~N} / \mathrm{cm} 2$
2200
4175
251124,7449
501,1234827
1,154118605

\subsection{Resistencia del Hilo de Fibra Fina y Fibra Gruesa}

Al aplicarse la prueba $T$ de student se pudo determinar que la fibra fina presentó una media de $590 \mathrm{~N} / \mathrm{cm} 2$ y la fibra gruesa 2835,5 N/cm2 observándose diferencias altamente significativas $(p \leq 0,01)$ en cuanto a la resistencia, la fibra fina presenta un rango promedio de resistencia de 862,5 \pm 250 mientras que la fibra gruesa fue de $4175 \pm 2200$ con una desviación estándar de 163,83 y 501,12 para fibra fina y gruesa respectivamente.

Valores que la ser comparados con los reportados por [13] que obtuvieron resultados de resistencia a la tensión de la fibra de alpaca de $2663.33 \mathrm{~N} / \mathrm{cm} 2$ pudiendo deberse estas diferencias a que aplicaron un lavado con sustancias químicas que posiblemente mejoraron la calidad de la fibra. Dicho valor se encuentra muy por encima del resultado obtenido en esta investigación. De acuerdo a lo establecido por la norma técnica IUP 6 , infiere como límites permisibles entre 800 a 1200 N/cm2.

\subsection{Porcentaje de Elongación}

Table 5

Porcentaje de elongación.

Datos
Media
Mínimo
Máximo
Varianza
Desviación estándar
Coeficiente de variación
Valor crítico de t (una cola)

Fibra fina
2,0906
1,17
3
0,161296
0,40161617375351
0,4596334
$2,70867397699393 E-16519$

Firba gruesa
1,236
0,8
1,5
0,032555
0,180430324615393
$-0,4582338$


Mediante la prueba $T$ de student se pudo determinar que la fibra fina presentó una media de $20 \%$ y la fibra gruesa con $12 \%$ presentan diferencias altamente significativas $(p \leq 0,01)$ de elongación, ya que la fibra fina presenta un rango promedio de resistencia de $3 \pm 1,17$ mientras que la fibra gruesa presenta un rango promedio de resistencia de 1,5 \pm 0,8 con una desviación estándar de 0,45 para la fibra fina y 0,18 para la fibra gruesa [13]. Mencionan que los resultados del porcentaje de elongación de la fibra de alpaca fueron de $53.50 \%$ y $47,50 \%$. Tomando en cuenta que dichos valores son diferentes al resultado que se obtuvo debido al tipo de lavado que realizaron en la investigación citada.

\subsection{Resistencia del Tejido con Fibra Fina y Fibra Gruesa}

Table 6

Prueba de lastometría.

\begin{tabular}{|l|l|l|l|l|l|}
\hline Tratamiento & Muestra no. & Tiempo (s) & BAR & PSI & Lastometría \\
\hline T1 Fibra fina & 1 & 1,88 & 1,7 & 25 & 9,87 \\
\hline T2 Fibra gruesa & 1 & 2,43 & 2,3 & 34 & 10,07 \\
\hline
\end{tabular}

La prueba de lastometría se realizó en un lastometro en el cual el tejido realizado con el hilado de la fibra gruesa tuvo mejor resistencia (2,3 BAR) que el tejido realizado con el hilado de la fibra fina (1,7 BAR), el hilo grueso resistió por más tiempo (2,34 s) a diferencia del hilo fino que resistió (1,88 s). Esta diferencia puede ser debida al tipo de tejido y al grosor del hilo.

\subsection{Pruebas de Suavidad al Tacto}

La evaluación sensorial de la suavidad de las fibras, permite clasificarlas con respecto a la finura, esta clasificación establece: fibras finas suaves y flexibles, de acuerdo a los datos obtenidos a la fibra de alpaca se la cataloga como una fibra fina, datos que lo presenta la Tabla 7.

Se realizó las pruebas a 11 evaluadores para determinar la calidad de la fibra de alpaca obteniendo como resultado que la fibra de alpaca se encuentra dentro de la categoría suave para ambos tipos de fibra.

Luego de la prueba de suavidad al tacto y de ordenamiento. El 100\% de los evaluadores indicaron que la fibra gruesa se encontraba dentro de la categoría suave.

De los 11 evaluadores el 91\% que corresponde a 10 evaluadores indicaron que la fibra fina de alpaca se encontraba dentro de la categoría suave, mientras que el $9 \%$ restante que corresponde a 1 evaluador indicó que la fibra fina se encontraba en la categoría extra suave. 
Table 7

Prueba de suavidad.

\begin{tabular}{l|l|l} 
No evaluadores & Fibra fina & Fibra gruesa \\
\hline Categoría & 1 & 0 \\
\hline Extra suave & 10 & 11 \\
\hline Suave & 0 & 0 \\
\hline Dura & 0 & 0 \\
\hline Extradura & 11 & 11 \\
\hline Total & $100 \%$ & $100 \%$ \\
\hline$\%$ & &
\end{tabular}

Figure 5
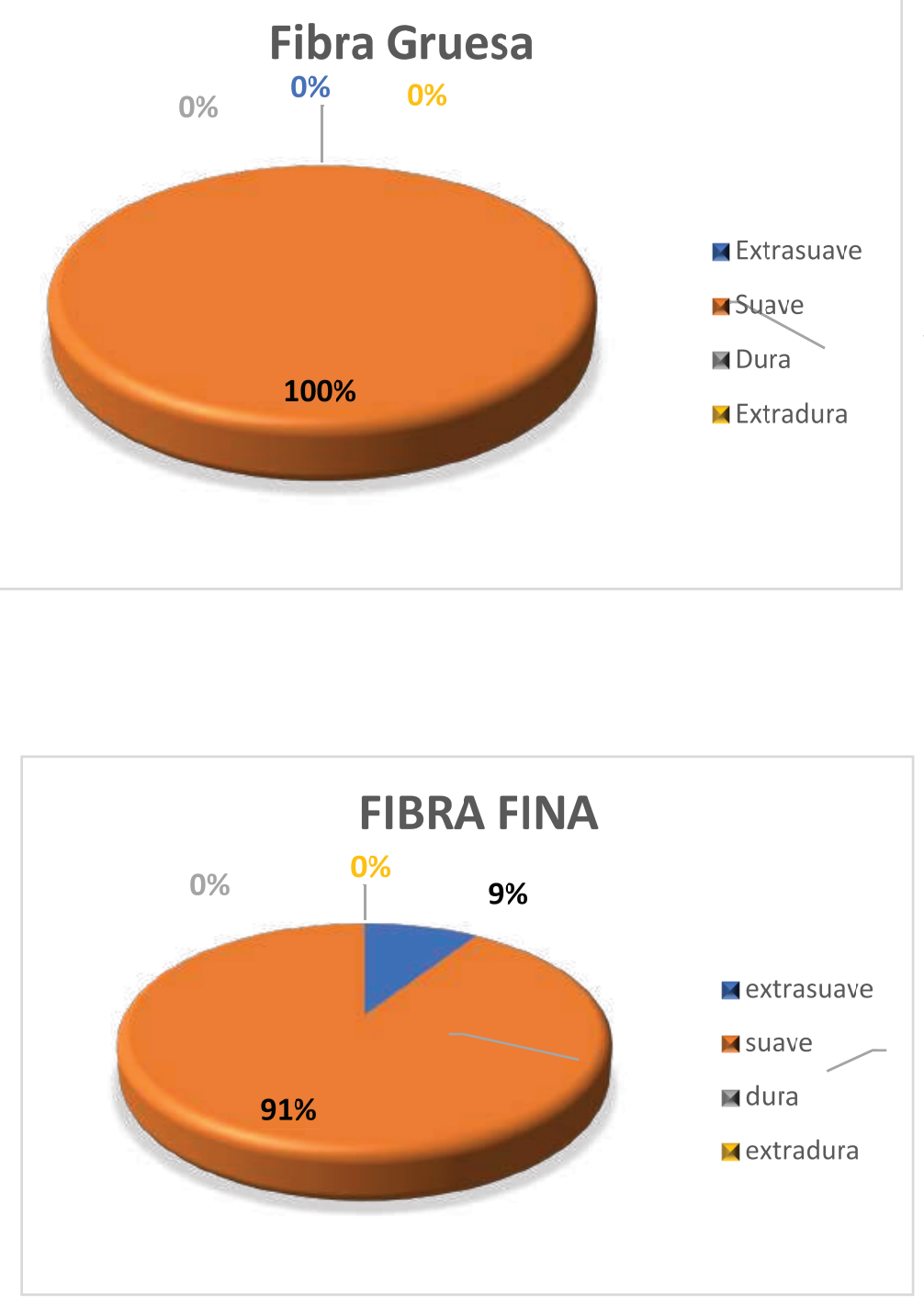

Figure 6 


\section{Conclusiones}

Luego de analizar las diferentes variables, las conclusiones que se extraen en la presente investigación sobre la de la fibra de alpaca son las siguientes:

- El rendimiento al lavado del vellón de la fibra de alpaca Huacaya fue de 85,71\%. En cuanto a la longitud de fibra sin estirar para fibra fina, su media fue de 12,50 $\mathrm{cm}$ y para la fibra gruesa de $13,52 \mathrm{~cm}$ a diferencia de la media estirada que presento de 17,29 y $17,27 \mathrm{~cm}$ para fibra fina y gruesa respectivamente.

- La resistencia a la tensión (N/cm2) para la fibra fina de alpaca fue de $590 \mathrm{~N} / \mathrm{cm} 2$ con un porcentaje de elongación de 2,09 y la fibra gruesa presento valores de $2835,5 \mathrm{~N} / \mathrm{cm} 2$ y de elongación de 1,23\%. Para la elastometría, la fibra gruesa presento mejores resultados 2,3 BAR a diferencia de la fibra fina que fue de 1,7 BAR.

- En las pruebas sensoriales de suavidad, los participantes categorizaron la fibra de alpaca Huacaya como una fibra suave con un $91 \%$ del total y un $9 \%$ la colocaron como una fibra extra suave.

\section{References}

[1] Valencia, F. E. (2017). La cadena de valor para optimizar la producción de fibra de Alpaca en la empresa Sais Sollocota Ltda. № 5 - Perú. scielo Perú. Obtenido de http: //www.scielo.org.pe/scielo.php?script=sci_arttext\&pid=S221971682017000200006

[2] INEI. (2012). IV CENSO NACIONAL AGROPECUARIO. PERÚ-LIMA: Ministerio de Agricultura y Riego. Obtenido de http://proyectos.inei.gob.pe/web/ DocumentosPublicos/ResultadosFinalesIVCENAGRO.pdf

[3] INEC. (2014). III CENSO NACIONAL AGROPECUARIO. QUITO- ECUADOR: SICA- MINISTERIO DE AGRICULTURA Y GANADERIA-. Obtenido de https: //www.ecuadorencifras.gob.ec/documentos/web-inec/Estadisticas_agropecuarias/ CNA/Tomo_CNA.pdf

[4] Natahaly, S. S. (2015). Latacunga, Cotopaxi, Ecuador. Obtenido de http://repositorio. utc.edu.ec/bitstream/27000/2824/1/T-UTC-00348.pdf

[5] Quispe Peña, E., Poma Gutiérrez, A., \& Purroy Unanua, A. (2013). CARACTERISTICAS PRODUCTIVAS Y TEXTILES DE LA FIBRA DE ALPACAS DE RAZA HUACAYA A REVIEW OF HUACAYA ALPACAS FIBER TRAITS. Revista Complutense de Ciencias Veterinarias, 7(1), 1-29.

[6] Ernesto Ccana, E. A. (2008). Técnicas apropiadas de esquila de alpaca. Cusco Perú: Biblioteca Nacional del Perú № 2008-16275. 
[7] Aylan-Parker, J., \& McGregor, B. A. (2002). Optimising sampling techniques and estimating sampling variance of fleece quality attributes in alpacas. Small Ruminant Research, 44(1), 53-64.

[8] INEN 2852.2015. norma Tecnica Ecuatoriana. Fibra de Alpaca en vellon. 2015.

[9] IWTO-19 (International Wool Textile Organization). 2012. Determination of Wool Base and Vegetable Matter Base of Core Samples of Raw Wool. International Wool Textile Organization Specification, The Woolmark Company, Ilkley, England.

[10] Wuliji, T., Davis, G. H., Dodds, K. G., Turner, P. R., Andrews, R. N., \& Bruce, G. D. (2000). Production performance, repeatability and heritability estimates for live weight, fleece weight and fiber characteristics of alpacas in New Zealand. Small Ruminant Research, 37(3), 189-201.

[11] McGregor, B. A. (2006). Production, attributes and relative value of alpaca fleeces in southern Australia and implications for industry development. Small Ruminant Research, 61(2-3), 93-111.

[12] Gamarra Sánchez, G. (2006). "CORRELACIÓN ENTRE EL NÚMERO DE RIZOS, DIÁMETRO DE FIBRA, LONGITUD DE MECHA Y DE FIBRA EN ALPACAS HEMBRAS HUACAYA EN LA UNIDAD DE PRODUCCIÓN COCHAS DE LA S.A.I.S. TUPAC AMARU LTDA. N ${ }^{\circ}$ 1. Obtenido de http://repositorio.uncp.edu.pe/bitstream/handle/ UNCP/2924/Gamarra\{\%\}20Sanchez.pdf?sequence=1\&isAllowed=y

[13] McMillan, J., \& Zoido, P. (2004). How to subvert democracy: Montesinos in Peru. Journal of Economic perspectives, 18(4), 69-92.

[14] CARPENTER, R., LYON, D., HASDELL, T. (2000) Análisis sensorial en el desarrollo y control de la calidad de alimentos. Editorial Acribia. Segunda edición. Zaragoza, España 2000.

[15] FAO. (13 de 11 de 2019). Obtenido de http://www.fao.org/ecuador/noticias/ detail-events/en/c/1115285/

[16] Wendy Huebla S., Jesica Rea R., 2019. tesis de grado como Ingenieros en industrias Pecuarias de la Escuela Superior Politécnica de Chimborazo. 